A Practical Comparison of Ligation-Independent Cloning Techniques Julian Stevenson ${ }^{1,3, *}$, James R. Krycer ${ }^{1,2,3, *}$, Lisa Phan ${ }^{1}$, and Andrew J. Brown ${ }^{1, *}$

${ }^{1}$ School of Biotechnology and Biomolecular Sciences, The University of New South Wales, Sydney, NSW, 2052, Australia

${ }^{2}$ Diabetes and Obesity Program, Garvan Institute of Medical Research, Sydney, NSW, 2010, Australia

${ }^{3}$ These authors contributed equally to this work

* To whom correspondence should be addressed. Tel: +61-2-9385-2005; Fax: +61-29385-1483; Email: aj.brown@unsw.edu.au

Correspondence may also be addressed to Julian Stevenson; Email:

j.stevenson@unsw.edu.au or James R. Krycer; Email: j.krycer@garvan.org.au

\title{
SUPPORTING FIGURE AND TABLES
}

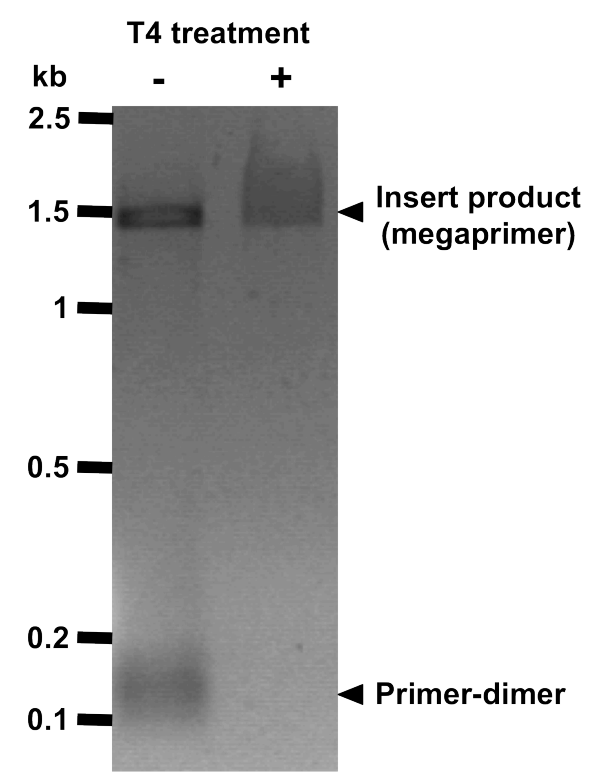

Figure S1. Primer-dimer can be removed with T4 DNA polymerase exonuclease treatment. $0.25 \mathrm{pmol}$ of $1.4 \mathrm{~kb}$ LXR purified PCR product was treated for $30 \mathrm{~min}$ at $25^{\circ} \mathrm{C}$ with $3 \mathrm{U}$ of T4 DNA polymerase. 
Table S1. Primer sequences. Vector tail sequences are indicated by boldface.

Overlapping reverse-complementary sequences are underlined.

\begin{tabular}{|c|c|}
\hline Primer Name & Primer Sequence $\left(5^{\prime} \rightarrow 3^{\prime}\right)$ \\
\hline KanR-in-pUC-F & $\begin{array}{l}\text { CGTGCCAGCTGCATTAATGAATCGGCCAACGCGGAACCCCTATTT } \\
\text { GTTTA }\end{array}$ \\
\hline KanR-in-pUC-R & $\begin{array}{l}\text { CGAGCGCAGCGAGTCAGTGAGCGAGGAAGCTCATTTCGAACCCCA } \\
\text { GAGTC }\end{array}$ \\
\hline pUC18-F & GCTTCCTCGCTCACTGACTC \\
\hline pUC18-R & GTTGGCCGATTCATTAATGC \\
\hline pUC18-L30-F & CGAGCTCGAATTCGTAATCATGGTCATAGC \\
\hline pUC18-L30-R & CGCATATGGTGCACTCTCAGTACAATCTGC \\
\hline FLAG-pUC18-F & $\begin{array}{l}\text { GACTACAAGGACGACGACGACAAACGAGCTCGAATTCGTAATCATG } \\
\text { GTCATAGC }\end{array}$ \\
\hline DUC18-R & $\begin{array}{l}\text { TTTGTCGTCGTCGTCCTTGTAGTCCGCATATGGTGCACTCTCAGTA } \\
\text { CAATCTGC }\end{array}$ \\
\hline $\begin{array}{l}\text { FLAG-pUC18- } \\
\text { overlap-F }\end{array}$ & $\begin{array}{l}\text { GCAGATTGTACTGAGAGTGCACCATATGCGGACTACAAGGACGAC } \\
\text { GACGACAAA }\end{array}$ \\
\hline $\begin{array}{l}\text { FLAG-pUC18- } \\
\text { overlap-R }\end{array}$ & $\begin{array}{l}\text { GCTATGACCATGATTACGAATTCGAGCTCGTTTGTCGTCGTCGTCC } \\
\text { TTGTAGTC }\end{array}$ \\
\hline LXRb-pUC18-F & $\begin{array}{l}\text { GCAGATTGTACTGAGAGTGCACCATATGCGTCCTCTCCTACCACGA } \\
\text { GTTC }\end{array}$ \\
\hline LXRb-pUC18-R & $\begin{array}{l}\text { GCTATGACCATGATTACGAATTCGAGCTCGCTCGTGGACGTCCCA } \\
\text { GATCT }\end{array}$ \\
\hline Gluc-pUC18-F & $\begin{array}{l}\text { GCAGATTGTACTGAGAGTGCACCATATGCGCAACGAAGACTTCAAC } \\
\text { ATCGTG }\end{array}$ \\
\hline BGH-pUC18-R & $\begin{array}{l}\text { GCTATGACCATGATTACGAATTCGAGCTCGTAGAAGGCACAGTCGA } \\
\text { GG }\end{array}$ \\
\hline T7-pUC18-F & $\begin{array}{l}\text { GCAGATTGTACTGAGAGTGCACCATATGCGCGCAAATGGGCGGTA } \\
\text { GGCGTG }\end{array}$ \\
\hline SRC1-pUC18-F & $\begin{array}{l}\text { GCAGATTGTACTGAGAGTGCACCATATGCGAGTGGCCTCGGGGAC } \\
\text { AGTT }\end{array}$ \\
\hline SRC1-pUC18-R & $\begin{array}{l}\text { GCTATGACCATGATTACGAATTCGAGCTCGTTCAGTCAGTAGCTGC } \\
\text { TGAAGGA }\end{array}$ \\
\hline AR-pUC18-F & $\begin{array}{l}\text { GCAGATTGTACTGAGAGTGCACCATATGCGATGGAAGTGCAGTTA } \\
\text { GGGCTGGGAA }\end{array}$ \\
\hline AR-pUC18-R & $\begin{array}{l}\text { GCTATGACCATGATTACGAATTCGAGCTCGCTGGGTGTGGAAATAG } \\
\text { ATGG }\end{array}$ \\
\hline
\end{tabular}


Table S2. Plasmid names and gene accession numbers. Gene sequences can be found on the NCBI GenBank or RefSeq databases. Vector backbone information is available on the Addgene Vector Database (http://www.addgene.org/vectordatabase/).

\begin{tabular}{|l|c|c|c|c|}
\hline \multicolumn{1}{|c|}{ Plasmid } & Gene & $\begin{array}{c}\text { NCBI Gene } \\
\text { Accession } \\
\text { Number }\end{array}$ & $\begin{array}{c}\text { Vector } \\
\text { Backbone }\end{array}$ & Reference \\
\cline { 1 - 3 } pTK-AR-V5 & AR & NM_000044.3 & $\begin{array}{l}\text { pcDNA3.1D/V5- } \\
\text { His-TOPO }\end{array}$ & {$[6]$} \\
\hline $\begin{array}{l}\text { FLAG-hLXRß- } \\
\text { hGluc(1) }\end{array}$ & hGluc(1) & HQ388295.1 & pCMX & {$[6]$} \\
\cline { 2 - 3 } $\begin{array}{l}\text { pCMV-Insig-1- } \\
\text { Myc }\end{array}$ & Insig-1 & NM_007121.5 & pcDNA3 & {$[8]$} \\
\hline pCMV-SCAP & SCAP & NM_012235.2 & pcDNA3 & {$[8]$} \\
\hline pCR3.1-SRC-1 & SRC-1 & NM_003743.4 & pCR3.1 & {$[7]$} \\
\hline pUC18/Kan & \multicolumn{2}{|c|}{$\begin{array}{l}\text { See supporting .txt file for complete vector } \\
\text { sequence and main text for plasmid map. }\end{array}$} & Current work \\
\hline
\end{tabular}

Table S3. Effect of template concentration on PIPE cloning efficiency. Vector products amplified from $5 \mathrm{ng}$ or $0.5 \mathrm{ng}$ of template per $50 \mu \mathrm{l}$ reaction were combined with purified insert at a $5: 1 \mathrm{I}: \mathrm{V}$ ratio. 40 colonies were patched onto kanamycin/Xgal/IPTG plates to screen for potential recombinants (white colonies), rather than empty vector (blue colonies) or insert vector (kanamycin sensitive, Kan (-), colonies). Cloning efficiency is the percentage of successful recombinants.

\begin{tabular}{|c|c|c|c|c|c|c|}
\hline Template & Insert & Colonies & $\begin{array}{c}\text { Cloning } \\
\text { Efficiency }\end{array}$ & White & Blue & Kan (-) \\
\hline \multirow{3}{*}{$5 \mathrm{ng}$} & $350 \mathrm{bp}$ & 2420 & $28 \%$ & 11 & 29 & 0 \\
\cline { 2 - 7 } & $1.4 \mathrm{~kb}$ & 1620 & $30 \%$ & 12 & 28 & 0 \\
\cline { 2 - 7 } & $4.3 \mathrm{~kb}$ & 1070 & $8 \%$ & 3 & 37 & 0 \\
\hline \multirow{3}{*}{$0.5 \mathrm{ng}$} & $350 \mathrm{bp}$ & 967 & $88 \%$ & 35 & 3 & 2 \\
\cline { 2 - 7 } & $1.4 \mathrm{~kb}$ & 814 & $93 \%$ & 37 & 2 & 1 \\
\cline { 2 - 7 } & $4.3 \mathrm{~kb}$ & 140 & $88 \%$ & 35 & 4 & 1 \\
\hline
\end{tabular}


Table S4. Cycle number for insert product does not affect PIPE cloning

efficiency. Insert products purified from 25-40 cycle reactions were combined at a 5:1

$\mathrm{I}: \mathrm{V}$ ratio with 40 cycle vector product.

\begin{tabular}{|c|c|c|c|c|c|c|c|}
\hline Insert & Exp. \# & Cycles & Colonies & $\begin{array}{l}\text { Cloning } \\
\text { Efficiency }\end{array}$ & White & Blue & Kan (-) \\
\hline \multirow{12}{*}{$350 \mathrm{bp}$} & \multirow[t]{4}{*}{1} & 25 & 1080 & $63 \%$ & 25 & 14 & 1 \\
\hline & & 30 & 830 & $78 \%$ & 31 & 9 & 0 \\
\hline & & 35 & 935 & $65 \%$ & 26 & 14 & 0 \\
\hline & & 40 & 880 & $68 \%$ & 27 & 13 & 0 \\
\hline & \multirow[t]{4}{*}{2} & 25 & 2220 & $83 \%$ & 33 & 5 & 2 \\
\hline & & 30 & 2610 & $73 \%$ & 29 & 10 & 1 \\
\hline & & 35 & 1860 & $60 \%$ & 24 & 13 & 2 \\
\hline & & 40 & 2600 & $78 \%$ & 31 & 8 & 1 \\
\hline & \multirow[t]{4}{*}{3} & 25 & 1550 & $50 \%$ & 20 & 24 & 0 \\
\hline & & 30 & 1450 & $58 \%$ & 23 & 19 & 0 \\
\hline & & 35 & 2430 & $73 \%$ & 29 & 11 & 0 \\
\hline & & 40 & 2200 & $63 \%$ & 25 & 15 & 0 \\
\hline \multirow{12}{*}{$1.4 \mathrm{~kb}$} & \multirow[t]{4}{*}{1} & 25 & 1200 & $80 \%$ & 32 & 8 & 0 \\
\hline & & 30 & 1510 & $93 \%$ & 37 & 3 & 0 \\
\hline & & 35 & 1510 & $78 \%$ & 31 & 9 & 0 \\
\hline & & 40 & 1230 & $88 \%$ & 35 & 5 & 0 \\
\hline & \multirow[t]{4}{*}{2} & 25 & 685 & $48 \%$ & 19 & 21 & 0 \\
\hline & & 30 & 830 & $48 \%$ & 19 & 20 & 1 \\
\hline & & 35 & 1130 & $68 \%$ & 27 & 12 & 1 \\
\hline & & 40 & 1520 & $78 \%$ & 31 & 9 & 0 \\
\hline & \multirow[t]{4}{*}{3} & 25 & 646 & $78 \%$ & 31 & 6 & 3 \\
\hline & & 30 & 1400 & $75 \%$ & 30 & 9 & 1 \\
\hline & & 35 & 1140 & $85 \%$ & 34 & 4 & 2 \\
\hline & & 40 & 1020 & $80 \%$ & 32 & 7 & 1 \\
\hline \multirow{12}{*}{$4.3 \mathrm{~kb}$} & \multirow{4}{*}{1} & 25 & 715 & $85 \%$ & 34 & 6 & 0 \\
\hline & & 30 & 600 & $80 \%$ & 32 & 8 & 0 \\
\hline & & 35 & 605 & $68 \%$ & 27 & 13 & 0 \\
\hline & & 40 & 600 & $75 \%$ & 30 & 10 & 0 \\
\hline & \multirow[t]{4}{*}{2} & 25 & 330 & $40 \%$ & 16 & 24 & 0 \\
\hline & & 30 & 357 & $45 \%$ & 18 & 22 & 0 \\
\hline & & 35 & 961 & $75 \%$ & 30 & 10 & 0 \\
\hline & & 40 & 1960 & $95 \%$ & 38 & 2 & 0 \\
\hline & \multirow[t]{4}{*}{3} & 25 & 655 & $85 \%$ & 34 & 2 & 4 \\
\hline & & 30 & 740 & $73 \%$ & 29 & 10 & 1 \\
\hline & & 35 & 860 & $78 \%$ & 31 & 8 & 1 \\
\hline & & 40 & 940 & $80 \%$ & 32 & 7 & 1 \\
\hline
\end{tabular}


Table S5. Cycle number for vector product does not affect PIPE cloning

efficiency. Vector products purified from 25-40 cycle reactions were combined at a 5:1 I:V ratio with 40 cycle insert products.

\begin{tabular}{|c|c|c|c|c|c|c|}
\hline Insert & Cycles & Colonies & $\begin{array}{c}\text { Cloning } \\
\text { Efficiency }\end{array}$ & White & Blue & Kan (-) \\
\hline \multirow{3}{*}{$1.4 \mathrm{~kb}$} & 25 & 2450 & $93 \%$ & 37 & 2 & 1 \\
\cline { 2 - 7 } & 30 & 2560 & $88 \%$ & 35 & 5 & 0 \\
\cline { 2 - 7 } & 35 & 3560 & $88 \%$ & 35 & 5 & 0 \\
\cline { 2 - 7 } & 40 & 3360 & $88 \%$ & 35 & 5 & 0 \\
\hline \multirow{3}{*}{$4.3 \mathrm{~kb}$} & 25 & 638 & $95 \%$ & 38 & 2 & 0 \\
\cline { 2 - 7 } & 30 & 660 & $88 \%$ & 35 & 5 & 0 \\
\cline { 2 - 7 } & 35 & 605 & $93 \%$ & 37 & 3 & 0 \\
\cline { 2 - 7 } & 40 & 412 & $75 \%$ & 30 & 10 & 0 \\
\hline
\end{tabular}

Table S6. Effect of insert:vector ratio on PIPE cloning efficiency. Purified products were combined at the indicated molar ratios.

\begin{tabular}{|c|c|c|c|c|c|c|c|}
\hline Insert & Exp. \# & $\mathrm{I}: \mathrm{V}$ & Colonies & $\begin{array}{l}\text { Cloning } \\
\text { Efficiency }\end{array}$ & White & Blue & Kan (-) \\
\hline \multirow{4}{*}{$350 \mathrm{bp}$} & \multirow[t]{4}{*}{1} & $1: 1$ & 585 & $40 \%$ & 16 & 24 & 0 \\
\hline & & $2.5: 1$ & 1180 & $75 \%$ & 30 & 9 & 1 \\
\hline & & $5: 1$ & 1250 & $80 \%$ & 32 & 8 & 0 \\
\hline & & $10: 1$ & 2240 & $90 \%$ & 36 & 4 & 0 \\
\hline \multirow{15}{*}{$1.4 \mathrm{~kb}$} & \multirow[t]{5}{*}{1} & $1: 1$ & 2700 & $68 \%$ & 27 & 13 & 0 \\
\hline & & $2.5: 1$ & 2490 & $90 \%$ & 36 & 4 & 0 \\
\hline & & $5: 1$ & 2420 & $90 \%$ & 36 & 4 & 0 \\
\hline & & $7.5: 1$ & 3380 & $90 \%$ & 36 & 4 & 0 \\
\hline & & $10: 1$ & 3290 & $93 \%$ & 37 & 3 & 0 \\
\hline & \multirow[t]{5}{*}{2} & $1: 1$ & 1600 & $90 \%$ & 36 & 4 & 0 \\
\hline & & $2.5: 1$ & 1980 & $93 \%$ & 37 & 3 & 0 \\
\hline & & $3: 1$ & 3300 & $83 \%$ & 33 & 3 & 0 \\
\hline & & $5: 1$ & 3170 & $88 \%$ & 35 & 5 & 0 \\
\hline & & $10: 1$ & 2400 & $85 \%$ & 34 & 6 & 0 \\
\hline & \multirow[t]{5}{*}{3} & $1: 1$ & 775 & $90 \%$ & 36 & 4 & 0 \\
\hline & & $2.5: 1$ & 1190 & $95 \%$ & 38 & 2 & 0 \\
\hline & & $5: 1$ & 785 & $100 \%$ & 40 & 0 & 0 \\
\hline & & $7.5: 1$ & 975 & $88 \%$ & 35 & 4 & 1 \\
\hline & & $10: 1$ & 720 & $80 \%$ & 32 & 8 & 0 \\
\hline \multirow{4}{*}{$4.3 \mathrm{~kb}$} & \multirow[t]{4}{*}{1} & $1: 1$ & 516 & $78 \%$ & 31 & 9 & 0 \\
\hline & & $2.5: 1$ & 715 & $90 \%$ & 36 & 4 & 0 \\
\hline & & $5: 1$ & 620 & $75 \%$ & 30 & 10 & 0 \\
\hline & & $10: 1$ & 500 & $85 \%$ & 34 & 6 & 0 \\
\hline
\end{tabular}


Table S7. Effect of T4 exonuclease treatment time on colony number. 0.025 pmol of vector and $0.0625 \mathrm{pmol}$ insert purified products were digested with DpnI then treated for 5 or $10 \mathrm{~min}$ with $0.75 \mathrm{U}$ of T4 DNA polymerase, as described in the Materials and Methods. Untreated (0 min) conditions are identical to PIPE cloning.

\begin{tabular}{|c|c|c|c|c|c|c|c|}
\hline Insert & Exp. \# & $\begin{array}{c}\mathrm{T} 4 \\
\text { treatment } \\
(\mathrm{min})\end{array}$ & Colonies & $\begin{array}{l}\text { Cloning } \\
\text { Efficiency }\end{array}$ & White & Blue & Kan (-) \\
\hline \multirow{3}{*}{$85 \mathrm{bp}$} & \multirow[t]{3}{*}{1} & 0 & 2650 & $98 \%$ & 39 & 0 & 1 \\
\hline & & 5 & 77700 & $100 \%$ & 40 & 0 & 0 \\
\hline & & 10 & 42000 & $100 \%$ & 40 & 0 & 0 \\
\hline \multirow{6}{*}{$350 \mathrm{bp}$} & \multirow[t]{3}{*}{1} & 0 & 176 & $98 \%$ & 39 & 1 & 0 \\
\hline & & 5 & 18500 & $98 \%$ & 39 & 0 & 1 \\
\hline & & 10 & 5440 & $100 \%$ & 40 & 0 & 0 \\
\hline & \multirow[t]{3}{*}{2} & 0 & 1040 & $91 \%$ & 19 & 1 & 1 \\
\hline & & 5 & 17000 & $100 \%$ & 40 & 0 & 0 \\
\hline & & 10 & 6300 & $100 \%$ & 40 & 0 & 0 \\
\hline \multirow{9}{*}{$1.4 \mathrm{~kb}$} & \multirow[t]{3}{*}{1} & 0 & 2420 & $100 \%$ & 40 & 0 & 0 \\
\hline & & 5 & 20000 & $98 \%$ & 39 & 0 & 1 \\
\hline & & 10 & 20000 & $100 \%$ & 40 & 0 & 0 \\
\hline & \multirow[t]{3}{*}{2} & 0 & 136 & $100 \%$ & 40 & 0 & 0 \\
\hline & & 5 & 8360 & $100 \%$ & 40 & 0 & 0 \\
\hline & & 10 & 8340 & $100 \%$ & 40 & 0 & 0 \\
\hline & \multirow[t]{3}{*}{3} & 0 & 483 & $100 \%$ & 40 & 0 & 0 \\
\hline & & 5 & 10200 & $100 \%$ & 40 & 0 & 0 \\
\hline & & 10 & 11400 & $100 \%$ & 40 & 0 & 0 \\
\hline \multirow{9}{*}{$4.3 \mathrm{~kb}$} & \multirow[t]{3}{*}{1} & 0 & 714 & $95 \%$ & 38 & 1 & 1 \\
\hline & & 5 & 13000 & $100 \%$ & 40 & 0 & 0 \\
\hline & & 10 & 23300 & $100 \%$ & 40 & 0 & 0 \\
\hline & \multirow[t]{3}{*}{2} & 0 & 474 & $100 \%$ & 40 & 0 & 0 \\
\hline & & 5 & 4150 & $98 \%$ & 39 & 1 & 0 \\
\hline & & 10 & 10700 & $95 \%$ & 38 & 2 & 0 \\
\hline & \multirow[t]{3}{*}{3} & 0 & 309 & $100 \%$ & 40 & 0 & 0 \\
\hline & & 5 & 6420 & $100 \%$ & 40 & 0 & 0 \\
\hline & & 10 & 2880 & $98 \%$ & 39 & 1 & 0 \\
\hline
\end{tabular}


Table S8. Effect of megaprimer concentration on overlap extension cloning

efficiency. $20 \mu \mathrm{L}$ OEC reactions were performed with $25 \mathrm{ng}$ template and $30^{\mathrm{a}}$ or $18^{\mathrm{b}}$ thermal cycles using the indicated amount of purified megaprimer. ${ }^{c}$ Anomalous result.

\begin{tabular}{|c|c|c|c|c|c|c|c|}
\hline $\begin{array}{l}\text { Mega- } \\
\text { primer }\end{array}$ & $\begin{array}{c}\text { Exp. } \\
\#\end{array}$ & fmol & Colonies & $\begin{array}{c}\text { Cloning } \\
\text { Efficiency }\end{array}$ & White & Blue & $\begin{array}{c}\text { Kan } \\
(-)\end{array}$ \\
\hline \multirow{10}{*}{$84 \mathrm{bp}$} & \multirow[t]{5}{*}{$1^{\mathrm{a}}$} & 100 & 2480 & $85 \%$ & 34 & 5 & 1 \\
\hline & & 250 & 15100 & $95 \%$ & 38 & 1 & 1 \\
\hline & & 500 & 11900 & $93 \%$ & 37 & 3 & 0 \\
\hline & & 750 & 1600 & $88 \%$ & 35 & 4 & 1 \\
\hline & & 1000 & 9300 & $88 \%$ & 35 & 5 & 0 \\
\hline & \multirow[t]{5}{*}{$2^{a}$} & 100 & 5690 & $70 \%$ & 28 & 12 & 0 \\
\hline & & 250 & 6380 & $93 \%$ & 37 & 3 & 0 \\
\hline & & 500 & 4710 & $88 \%$ & 35 & 5 & 0 \\
\hline & & 750 & 3470 & $80 \%$ & 32 & 8 & 0 \\
\hline & & 1000 & 1730 & $80 \%$ & 32 & 7 & 1 \\
\hline \multirow{22}{*}{$\begin{array}{l}350 \\
b p\end{array}$} & \multirow[t]{4}{*}{$1^{b}$} & 10 & 299 & $23 \%$ & 9 & 31 & 0 \\
\hline & & 33 & 2420 & $45 \%$ & 18 & 22 & 0 \\
\hline & & 100 & 3330 & $53 \%$ & 21 & 18 & 1 \\
\hline & & 330 & 7640 & $70 \%$ & 28 & 12 & 0 \\
\hline & \multirow[t]{6}{*}{$2^{b}$} & 10 & 525 & $15 \%$ & 6 & 33 & 1 \\
\hline & & 33 & 2400 & $38 \%$ & 15 & 24 & 1 \\
\hline & & 100 & 7720 & $45 \%$ & 18 & 19 & 3 \\
\hline & & 330 & 4410 & $43 \%$ & 17 & 13 & 10 \\
\hline & & 1000 & 3870 & $10 \%$ & 4 & 2 & 34 \\
\hline & & 3330 & 62 & $5 \%$ & 2 & 15 & 23 \\
\hline & \multirow[t]{6}{*}{$3^{b}$} & 10 & 89 & $30 \%$ & 12 & 27 & 1 \\
\hline & & 33 & 473 & $58 \%$ & 23 & 17 & 0 \\
\hline & & $100^{c}$ & 42 & $10 \%$ & 4 & 36 & 0 \\
\hline & & 330 & 9840 & $63 \%$ & 25 & 14 & 1 \\
\hline & & 1000 & 4440 & $20 \%$ & 8 & 15 & 17 \\
\hline & & 3330 & 51 & $10 \%$ & 4 & 7 & 29 \\
\hline & \multirow[t]{6}{*}{$4^{b}$} & 10 & 331 & $20 \%$ & 8 & 32 & 0 \\
\hline & & 33 & 3360 & $40 \%$ & 16 & 23 & 1 \\
\hline & & 100 & 10900 & $65 \%$ & 26 & 12 & 2 \\
\hline & & 330 & 11600 & $70 \%$ & 28 & 12 & 0 \\
\hline & & 1000 & 1240 & $38 \%$ & 15 & 21 & 4 \\
\hline & & 3330 & 10 & $10 \%$ & 1 & 9 & 0 \\
\hline \multirow{8}{*}{$1.4 \mathrm{~kb}$} & \multirow[t]{4}{*}{$1^{a}$} & 10 & 306 & $3 \%$ & 1 & 37 & 2 \\
\hline & & 25 & 408 & $18 \%$ & 7 & 33 & 0 \\
\hline & & 50 & 861 & $65 \%$ & 26 & 14 & 0 \\
\hline & & 100 & 1450 & $73 \%$ & 29 & 11 & 0 \\
\hline & \multirow[t]{4}{*}{$2^{a}$} & 10 & 145 & $20 \%$ & 8 & 32 & 0 \\
\hline & & 25 & 19 & $24 \%$ & 4 & 13 & 0 \\
\hline & & 50 & 13 & $15 \%$ & 2 & 11 & 0 \\
\hline & & 100 & 0 & $n / a$ & $\mathrm{n} / \mathrm{a}$ & $\mathrm{n} / \mathrm{a}$ & $\mathrm{n} / \mathrm{a}$ \\
\hline
\end{tabular}




\begin{tabular}{|c|c|c|c|c|c|c|c|}
\hline \multirow{3}{*}{$4.3 \mathrm{~kb}$} & $1^{\mathrm{a}}$ & 10 & 251 & $45 \%$ & 18 & 22 & 0 \\
\cline { 3 - 8 } & & 25 & 135 & $35 \%$ & 14 & 26 & 0 \\
\cline { 3 - 8 } & 50 & 20 & $21 \%$ & 5 & 18 & 1 \\
\cline { 3 - 8 } & 100 & 0 & $\mathrm{n} / \mathrm{a}$ & $\mathrm{n} / \mathrm{a}$ & $\mathrm{n} / \mathrm{a}$ & $\mathrm{n} / \mathrm{a}$ \\
\hline & $2^{\mathrm{a}}$ & 10 & 211 & $13 \%$ & 5 & 37 & 1 \\
\hline & 25 & 151 & $5 \%$ & 2 & 38 & 0 \\
\cline { 3 - 8 } & & 50 & 57 & 0 & 0 & 39 & 1 \\
\hline & 100 & 3 & 0 & 0 & 3 & 0 \\
\hline
\end{tabular}

Table S9. Increased PCR template can generate insert template background in OEC for high megaprimer concentrations. $20 \mu \mathrm{L}$ OEC reactions were performed with $25 \mathrm{ng}$ template and 18 thermal cycles using the indicated amount of purified 350 bp Gluc megaprimer obtained from a PCR using 5 or $0.5 \mathrm{ng}$ of template.

\begin{tabular}{|c|c|c|c|c|c|c|}
\hline $\begin{array}{c}\text { PCR } \\
\text { template }\end{array}$ & fmol & Colonies & $\begin{array}{c}\text { Cloning } \\
\text { Efficiency }\end{array}$ & White & Blue & Kan (-) \\
\hline \multirow{3}{*}{$5 \mathrm{ng}$} & 10 & 532 & $40 \%$ & 16 & 24 & 0 \\
\cline { 2 - 7 } & 33 & 1920 & $38 \%$ & 15 & 25 & 0 \\
\cline { 2 - 7 } & 100 & 3800 & $58 \%$ & 23 & 12 & 5 \\
\cline { 2 - 7 } & 330 & 2940 & $50 \%$ & 20 & 12 & 8 \\
\cline { 2 - 7 } & 1000 & 2670 & $15 \%$ & 6 & 6 & 28 \\
\cline { 2 - 7 } & 3330 & 19 & $0 \%$ & 0 & 6 & 13 \\
\hline \multirow{4}{*}{$0.5 \mathrm{ng}$} & 10 & 557 & $18 \%$ & 7 & 33 & 0 \\
\cline { 2 - 7 } & 33 & 2600 & $43 \%$ & 17 & 23 & 0 \\
\cline { 2 - 7 } & 100 & 5010 & $60 \%$ & 24 & 15 & 1 \\
\cline { 2 - 7 } & 330 & 2830 & $48 \%$ & 19 & 20 & 1 \\
\cline { 2 - 7 } & 1000 & 408 & $40 \%$ & 16 & 16 & 8 \\
\cline { 2 - 7 } & 3330 & 0 & $\mathrm{n} / \mathrm{a}$ & $\mathrm{n} / \mathrm{a}$ & $\mathrm{n} / \mathrm{a}$ & $\mathrm{n} / \mathrm{a}$ \\
\hline
\end{tabular}


Table S10. Colony number increases with additional cycles of overlap extension. $20 \mu \mathrm{L}$ OEC reactions were performed with $25 \mathrm{ng}$ template, $100 \mathrm{fmol}$ of purified 350 bp Gluc megaprimer and the indicated number of thermal cycles.

\begin{tabular}{|c|c|c|c|c|c|c|}
\hline Exp. \# & Cycles & Colonies & $\begin{array}{c}\text { Cloning } \\
\text { Efficiency }\end{array}$ & White & Blue & Kan (-) \\
\hline \multirow{4}{*}{1} & 14 & 3380 & $83 \%$ & 33 & 7 & 0 \\
\cline { 2 - 7 } & 16 & 5170 & $58 \%$ & 23 & 17 & 0 \\
\cline { 2 - 7 } & 18 & 9550 & $73 \%$ & 29 & 11 & 0 \\
\cline { 2 - 7 } & 20 & 13100 & $73 \%$ & 29 & 11 & 0 \\
\cline { 2 - 7 } & 22 & 15100 & $80 \%$ & 32 & 6 & 2 \\
\hline \multirow{6}{*}{2} & 14 & 4740 & $40 \%$ & 16 & 24 & 0 \\
\cline { 2 - 7 } & 18 & 8320 & $53 \%$ & 21 & 18 & 1 \\
\cline { 2 - 7 } & 22 & 14400 & $60 \%$ & 24 & 14 & 2 \\
\cline { 2 - 7 } & 26 & 28700 & $65 \%$ & 26 & 12 & 2 \\
\cline { 2 - 7 } & 30 & 33200 & $78 \%$ & 31 & 6 & 3 \\
\hline \multirow{6}{*}{3} & 14 & 2880 & $38 \%$ & 15 & 23 & 2 \\
\cline { 2 - 7 } & 18 & 6700 & $53 \%$ & 21 & 18 & 1 \\
\cline { 2 - 7 } & 22 & 12800 & $58 \%$ & 23 & 16 & 1 \\
\cline { 2 - 7 } & 26 & 15800 & $65 \%$ & 26 & 14 & 0 \\
\cline { 2 - 7 } & 30 & 21500 & $78 \%$ & 31 & 9 & 0 \\
\hline
\end{tabular}


Table S11. Direct comparison of PIPE, SLIC and OEC for increasing insert sizes. Techniques were performed using the optimised conditions, as described in the Materials and Methods.

\begin{tabular}{|c|c|c|c|c|c|c|c|}
\hline Insert & Exp. \# & Technique & Colonies & $\begin{array}{l}\text { Cloning } \\
\text { Efficiency }\end{array}$ & White & Blue & Dead \\
\hline \multirow{9}{*}{85 bp } & \multirow[t]{3}{*}{1} & PIPE & 1680 & $100 \%$ & 40 & 0 & 0 \\
\hline & & SLIC & 15100 & $100 \%$ & 40 & 0 & 0 \\
\hline & & OEC & 15100 & $95 \%$ & 38 & 1 & 1 \\
\hline & \multirow[t]{3}{*}{2} & PIPE & 806 & $100 \%$ & 40 & 0 & 0 \\
\hline & & SLIC & 8740 & $100 \%$ & 40 & 0 & 0 \\
\hline & & OEC & 6700 & $100 \%$ & 40 & 0 & 0 \\
\hline & \multirow[t]{3}{*}{3} & PIPE & 2650 & $98 \%$ & 39 & 0 & 1 \\
\hline & & SLIC & 77700 & $100 \%$ & 40 & 0 & 0 \\
\hline & & OEC & 10600 & $93 \%$ & 37 & 3 & 0 \\
\hline \multirow{12}{*}{$350 \mathrm{bp}$} & \multirow[t]{3}{*}{1} & PIPE & 284 & $93 \%$ & 37 & 2 & 1 \\
\hline & & SLIC & 1440 & $98 \%$ & 39 & 1 & 0 \\
\hline & & OEC & 15800 & $85 \%$ & 34 & 6 & 0 \\
\hline & \multirow[t]{3}{*}{2} & PIPE & 262 & $95 \%$ & 38 & 1 & 1 \\
\hline & & SLIC & 1870 & $98 \%$ & 39 & 0 & 1 \\
\hline & & OEC & 11400 & $78 \%$ & 31 & 9 & 0 \\
\hline & \multirow[t]{3}{*}{3} & PIPE & 1030 & $91 \%$ & 19 & 1 & 1 \\
\hline & & SLIC & 17000 & $100 \%$ & 40 & 0 & 0 \\
\hline & & OEC & 6970 & $90 \%$ & 36 & 0 & 4 \\
\hline & \multirow[t]{3}{*}{4} & PIPE & 176 & $98 \%$ & 39 & 1 & 0 \\
\hline & & SLIC & 18500 & $98 \%$ & 39 & 0 & 1 \\
\hline & & OEC & 9200 & $90 \%$ & 36 & 1 & 3 \\
\hline \multirow{6}{*}{$1.4 \mathrm{~kb}$} & \multirow[t]{3}{*}{1} & PIPE & 705 & $95 \%$ & 38 & 0 & 2 \\
\hline & & SLIC & 2760 & $100 \%$ & 40 & 0 & 0 \\
\hline & & OEC & 1450 & $73 \%$ & 29 & 11 & 0 \\
\hline & \multirow[t]{3}{*}{2} & PIPE & 183 & $100 \%$ & 40 & 0 & 0 \\
\hline & & SLIC & 1580 & $100 \%$ & 40 & 0 & 0 \\
\hline & & OEC & 145 & $20 \%$ & 8 & 32 & 0 \\
\hline \multirow{6}{*}{$4.3 \mathrm{~kb}$} & \multirow[t]{3}{*}{1} & PIPE & 309 & $100 \%$ & 40 & 0 & 0 \\
\hline & & SLIC & 6420 & $100 \%$ & 40 & 0 & 0 \\
\hline & & OEC & 251 & $45 \%$ & 18 & 22 & 0 \\
\hline & \multirow[t]{3}{*}{2} & PIPE & 110 & $95 \%$ & 38 & 2 & 0 \\
\hline & & SLIC & 1640 & $100 \%$ & 40 & 0 & 0 \\
\hline & & OEC & 211 & $13 \%$ & 5 & 37 & 1 \\
\hline
\end{tabular}


Table S12. Cloning of a 350 bp fragment without quantification or purification. $5 \mathrm{ng}$ of pUC18/Kan pre-cleaved with PstI was amplified by PCR.

${ }^{\mathrm{a}} 5 \mu \mathrm{L}$ of $350 \mathrm{bp}$ Gluc insert and vector PCR products were combined and digested with DpnI without dilution or purification.

${ }^{\mathrm{b}} 30 \mu \mathrm{L}$ of insert and vector PCR product were combined, purified and equal volumes treated with T4 for SLIC or left untreated for PIPE.

${ }^{\mathrm{c}} 1 \mu \mathrm{L}$ of unpurified insert PCR product was used as megaprimer for OEC.

${ }^{\mathrm{d}} 100 \mathrm{fmol}$ of purified megaprimer was used for OEC.

${ }^{\mathrm{e}}$ The number of colonies relative to unpurified, unconcentrated PIPE.

\begin{tabular}{|c|c|c|c|}
\hline Cloning Technique & $\begin{array}{l}\text { Cloning } \\
\text { Efficiency }\end{array}$ & Colonies & $\begin{array}{c}\text { Fold }^{\mathrm{e}} \\
\text { Increase }\end{array}$ \\
\hline PIPE $^{a}$ (Unpurified) & $95 \%$ & 226 & 1 \\
\hline PIPE $^{b}$ (Purified) & $95 \%$ & 631 & 3 \\
\hline $\mathrm{SLIC}^{\mathrm{b}}(5 \mathrm{~min})$ & $100 \%$ & 1920 & 8 \\
\hline $\mathrm{SLIC}^{\mathrm{b}}(10 \mathrm{~min})$ & $100 \%$ & 2220 & 10 \\
\hline OEC $^{\mathrm{C}}$ (Unpurified) & $80 \%$ & 44 & 0.2 \\
\hline OEC $^{d}$ (Purified) & $76 \%$ & 206 & 1 \\
\hline
\end{tabular}

